\title{
The comparison of brain stem transcriptomes in rats from hypertensive ISIAH and normotensive WAG strains
}

\author{
L.A. Fedoseeva ${ }^{1}$, L.O. Klimov ${ }^{1,2}$, N.I. Ershov ${ }^{1}$, V.M. Efimov ${ }^{1,2}$, A.L. Markel $^{1,2}$, \\ Y.L. Orlov ${ }^{1,2}$, O.E. Redina ${ }^{1 *}$ \\ ${ }^{1}$ Institute of Cytology and Genetics SB RAS, Novosibirsk, Russia \\ ${ }^{2}$ Novosibirsk State University, Novosibirsk, Russia \\ *e-mail:oredina@ngs.ru
}

Key words: stress-sensitive hypertension, brain stem, transcriptional profiling, RNA-Seq, ISIAH rats

Motivation and Aim: Neurons of the brain stem control the activity of the sympathetic nervous system and play an important role in the blood pressure (BP) regulation. The purpose of this work was to elucidate the central mechanisms that contribute to the genetic predisposition for the development of a stress-sensitive form of hypertension in ISIAH rats.

Methods: Using the RNA-Seq method, a comparative analysis of transcriptomes of the brain stems (medulla oblongata and pons) of hypertensive ISIAH rats and normotensive WAG rats at the age of 3 months was carried out. To identify the differentially expressed genes (DEGs), the Cufflinks / Cuffdiff software package was used, the PLS-DA (partialleast squares discriminant analysis) was employed to detect the DEGs, which may contribute the most to the inter-strain differences.

Results: A total of 224 DEGs were found in the brain stems of ISIAH and WAG rats. Their annotation in the databases made it possible to identify genes associated with hypertension, as well as with diseases of the central nervous system and with a number of diseases that often accompany the development of hypertension (immune system diseases, insulin resistance, diabetes). Functional annotation of DEGs has revealed a number of biological processes that can influence the development of arterial hypertension in ISIAH rats. The most statistically significant was the group of DEGs described by the term 'hormone metabolic process', which includes several genes that control the biosynthesis of steroid hormones. The most essential for the present work were the groups of genes described by terms 'blood circulation', 'regulation of blood pressure', 'regulation of blood vessel size', 'muscle contraction' and 'tissue remodeling'. The detection of groups of genes associated with a response to various stimuli, such as 'response to endogenous (hormone) stimulus', 'response to external stimulus', and 'response to stress' (oxidative stress) underlines the existence of the homeostatic problems in the brain stem of ISIAH rats. Using the PLS-DA method, the genes possibly making the maximum contribution to inter-strain differences have been identified.

Conclusion: The results obtained contribute to an understanding of the central mechanisms of genetic predisposition to the development of a stress-sensitive form of hypertension. Acknowledgements: The study has been supported by Russian Science Foundation and budget project No. 0324-2018-0016. 